\title{
含噻唑环结构的新型吡唑酰胺类化合物的合成与杀虫活性研究
}

\author{
梁 凯 ${ }^{\dagger}, a$ 周 钱 ${ }^{\dagger}, a$ 荀 校 ${ }^{a}$ 倪亚丹 ${ }^{a}$ 李金峰 ${ }^{a}$ 石玉军 $* a$ \\ 周环宇 ${ }^{a}$ 吴新星 $* a$ 石 健 ${ }^{b}$ 高 䂞 ${ }^{a}$ 戴 红*,a \\ ( ${ }^{a}$ 南通大学化学化工学院 江苏南通 226019) \\ ( ${ }^{b}$ 南通大学分析测试中心 江苏南通 226019)
}

\begin{abstract}
摘要 为了探索新的吡唑酰胺活性物质, 基于吡螨胺的结构, 将噻唑环通过酰胺键同吡唑环相连, 合成了 18 个见文 献报道的吡唑酰胺类化合物, 通过 ${ }^{1} \mathrm{H} \mathrm{NMR},{ }^{13} \mathrm{C} \mathrm{NMR}$ 和元素分析等手段确证了其结构. 生物活性测试研究显示, 所有 目标化合物在 $500 \mu \mathrm{g} / \mathrm{mL}$ 时对粘虫都有 $100 \%$ 的杀虫活性. 7 个目标化合物在 $500 \mu \mathrm{g} / \mathrm{mL}$ 时对蚜虫具有 $70 \% \sim 100 \%$ 的防 治效果. 同时, 3 个目标化合物在 $500 \mu \mathrm{g} / \mathrm{mL}$ 时对朱砂叶螨显示出 $40 \% \sim 50 \%$ 的防效.
\end{abstract}

关键词 噻唑; 吡唑酰胺; 合成; 杀虫活性

\section{Synthesis and Insecticidal Activities of Novel Pyrazole Amide Derivatives Containing a Thiazole Unit}

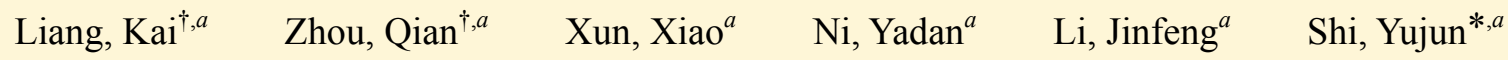 \\ Zhou, Huanyu $^{a} \quad \mathrm{Wu}, \mathrm{Xinxing}^{*, a} \quad \mathrm{Shi}$ Jian $^{b} \quad$ Gao, Lei $^{a} \quad$ Dai, Hong $^{*, a}$ \\ ( ${ }^{a}$ College of Chemistry and Chemical Engineering, Nantong University, Nantong, Jiangshu 226019) \\ ( ${ }^{b}$ Analysis and Testing Center, Nantong University, Nantong, Jiangshu 226019)
}

\begin{abstract}
In search of novel pyrazole amide compounds possessing good biological activities, a series of pyrazole amides were synthesized by introducing thiazole unit into pyrazole ring via amido bond, based on the lead of tebufenpyrad. The title compounds were structurually confirmed by ${ }^{1} \mathrm{H}$ NMR, ${ }^{13} \mathrm{C}$ NMR and elemental analysis. Preliminary bioassay displayed that all the title compounds exhibited $100 \%$ insecticidal activities against Oriental armyworm at $500 \mu \mathrm{g} / \mathrm{mL}$. At the concentration of $500 \mu \mathrm{g} / \mathrm{mL}$, seven compounds showed $70 \% \sim 100 \%$ mortality rate against Aphis medicaginis. In addition, three compounds had $40 \% \sim 50 \%$ insecticidal activity against Tetranychus cinnabarinus at $500 \mu \mathrm{g} / \mathrm{mL}$.

Keywords thiazole; pyrazole amide; synthesis; insecticidal activity
\end{abstract}

当前，含氮杂环衍生物因其具有结构多样性、生物 活性广谱等特点而成为新药创制的热点领域之一 ${ }^{[1-8]}$. 噻唑环作为含氮杂环化合物家族中的重要一员, 在农业 生产和医疗保健方面起到十分重要的作用, 常常被用于 杀虫、除草、杀菌、抗植物病毒、抗肿瘤等活性分子的 研究 ${ }^{[9 \sim 13]}$. 如诺华公司成功开发的噻虫嗪(Thiamethoxam)对蚜虫、叶蝉、粉禹、线虫等害虫有着优异的防治 效果 ${ }^{[14]}, \mathrm{CaO}$ 等 ${ }^{[15]}$ 报道新型含噻唑基丙烯腈衍生物 $\mathbf{A}$ 在 $50 \mathrm{mg} / \mathrm{L}$ 浓度下对蚜虫的杀灭活性为 $100 \%$, Ding 等 ${ }^{[16]}$
研究发现化合物 B 在 500 和 $100 \mu \mathrm{g} / \mathrm{mL}$ 浓度下对粘虫分 别有 $100 \%$ 和 $80 \%$ 的杀灭效果; Tan 等 ${ }^{[17]}$ 报道的化合物 $\mathbf{C}$ 在 $100 \mu \mathrm{g} / \mathrm{mL}$ 浓度下对马铃薯晚疫病和苹果褐斑病呈现 出良好的杀菌作用，其抑制率分别为 70\%和 $60 \%$. 吡唑 及其衍生物也是重要的一类含氮杂环, 因其优良的杀

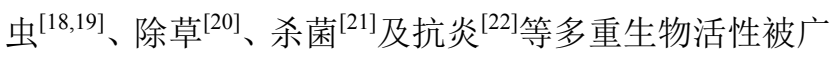
泛应用于农药、医药等领域. 目前已有许多具有广谱生 物活性的吡唑化合物被成功开发出来, 如日本三菱化学 公司研发的唑虫酰胺(Tolfenpyrad)与吡螨胺(Tebufen-

\footnotetext{
* Corresponding authors. E-mail: yjshi2015@163.com; wuxinxng@163.com; daihong_2015@aliyun.com Received January 22, 2020; revised February 25, 2020; published online February 29, 2020

Project supported by the National Natural Science Foundation of China (No. 21372135), the Science and Technology Project Fund of Nantong City (No. MS12019060) and the Program of High-level Talents of Nantong University (No. 03083031).

国家自然科学基金(Nos. 21372135)、南通市科技计划(No. MS12019060)和南通大学高层次人才(No. 03083031)资助项目.

共同第一作者(These authors contributed equally to this work).
} 
pyrad)对小菜蛾、附线螨等害虫表现优良的杀虫杀螨作

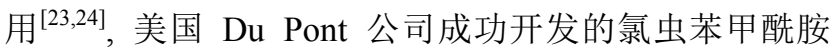
(Chlorantraniliprole) 对粘虫、蚜虫、叶蝉等害虫呈现出 较好的防治效果 ${ }^{[25]}$, 日本 Nihon Nohyaku 公司研制的唑 螨酯(Fenpyroximate) 对附线螨、红蜘蛛、植食性螨等害 虫具有优异杀灭活性 ${ }^{[26]}$. 此外, 酰胺基团也是十分常见 的结构骨架, 往往被选作关键药效团引入化合物分子结 构中来改善相应生物活性 ${ }^{[27 ~ 29]}$. 生物电子等排原理是 探索与发现先导药物的重要途径之一, 在新药创制研究 中具有广泛的应用 ${ }^{[30,31]}$. 近年来, 噻唑环作为苯环、吡 啶环等结构的电子等排体, 在生物活性分子的结构衍生 与优化方面发挥着重要作用 ${ }^{[32 ~ 34]}$. 如 Yang 等 ${ }^{[35]}$ 以杀螨 剂唑螨酯为先导化合物, 选用噻唑环代替唑螨酯结构中 的取代苯环部分, 合成得到的化合物 $\mathbf{D}$ 也展现出优良的 杀虫活性, 化合物 D 在 $5 \mathrm{mg} / \mathrm{L}$ 浓度下对蚊虫的杀灭效 果为 $100 \%$, 在 $200 \mathrm{mg} / \mathrm{L}$ 浓度下对蚜虫和螨虫的防效分 别为 $100 \%$ 和 $95 \%$. 因此, 为了进一步从吡唑类衍生物 中寻找与发现具有良好生物活性的化合物, 本研究以商 品化杀螨剂吡螨胺为农药先导, 通过生物电子等排法, 用取代的噻唑环替代吡螨胺分子结构中的取代苯环部
分, 将噻唑单元通过酰胺基团与吡唑环相连，设计并合 成出了一系列新型吡唑酰胺类化合物，同时评价其对粘 虫、螨虫和蚜虫的生物活性. 具体合成路线见 Scheme 1.

\section{1 结果与讨论}

\section{1 化合物的合成}

化合物 8 是制备目标化合物 12 的一个关键中间体， 选取 $\mathrm{R}^{2}$ 为 4- $\mathrm{Cl}$ 取代物为例，以中间体 7 为原料，尝试了 不同的还原剂、投料比以及反应溶剂对化合物 $\mathbf{8}$ 转化率 的影响. 从表 1 中可以看出, 当选以嗍氢化钠作还原剂, 乙醇为反应溶剂，中间体 7 与还原剂的投料比为 $1: 4$ 时，中间体 8 的合成收率相对较高，达到 $78 \%$.

选取目标化合物 $\mathbf{1 2 b}$ 为例，探索了不同的缚酸剂、 投料比、反应溶剂等反应条件对目标化合物 $12 \mathrm{~b}$ 单步合 成收率的影响. 由表 2 中可以看出, 当选用三乙胺为缚 酸剂，二氯甲烷为反应溶剂，化合物 11 与酰氯中间体 $\mathbf{5}$ 的投料比为 $1: 1.2$ 时，目标化合物 12b 的合成收率相对 较好，达到 $54 \%$. 最终通过该方法顺利制备出了 18 个含 噻唑环结构的新型吡唑酰胺类化合物.<smiles>CN1COCN(Cc2cnc(Cl)s2)C1=NO</smiles>

Thiamethoxam<smiles>CCCSC(C)C(=O)O/C(=C(/C#N)c1csc(-c2ccccc2)n1)c1cnc(C)s1</smiles>

A<smiles>[R]CC1CCN(C(=O)N2CCN(C)CC2)CC1c1nc(C)c(-c2cccc(Cl)c2)s1</smiles>

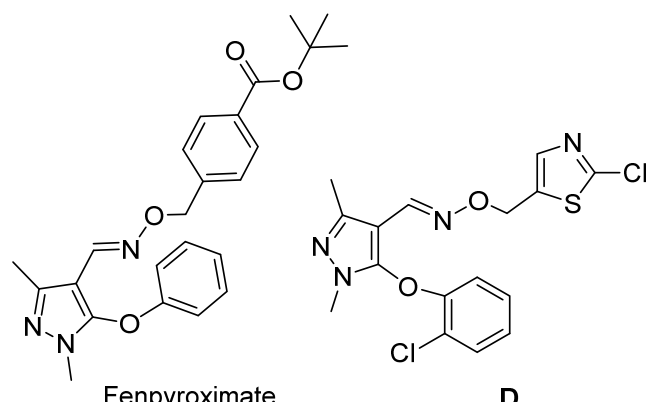

Tolfenpyrad

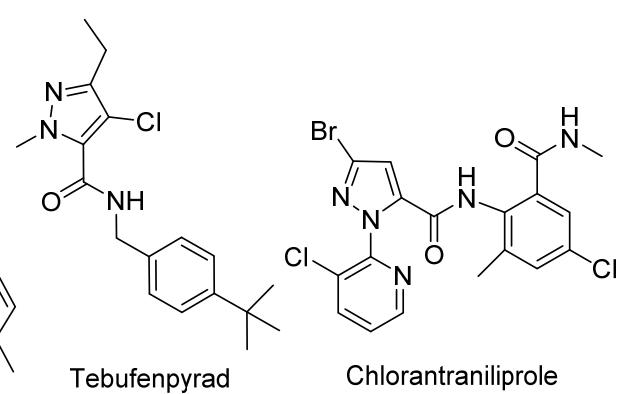

Fenpyroximate

图 1 化合物 $\mathbf{A} \sim \mathbf{D}$ 、噻虫嗪、唑虫酰胺、吡螨胺、氯虫苯甲酰胺和唑螨酯的化学结构

Figure 1 Chemical structures of compounds $\mathbf{A} \sim \mathbf{D}$, thiamethoxam, tolfenpyrad, tebufenpyrad and chlorantraniliprole and fenpyroximate
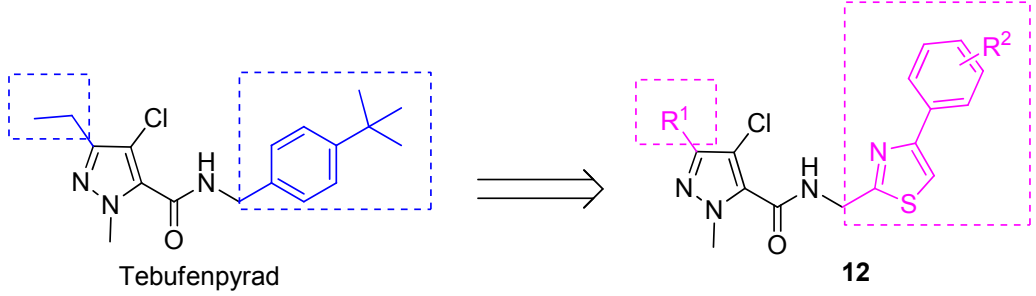

图 2 目标化合物 $\mathbf{1 2}$ 的分子设计

Figure 2 Design of the title compounds 12 

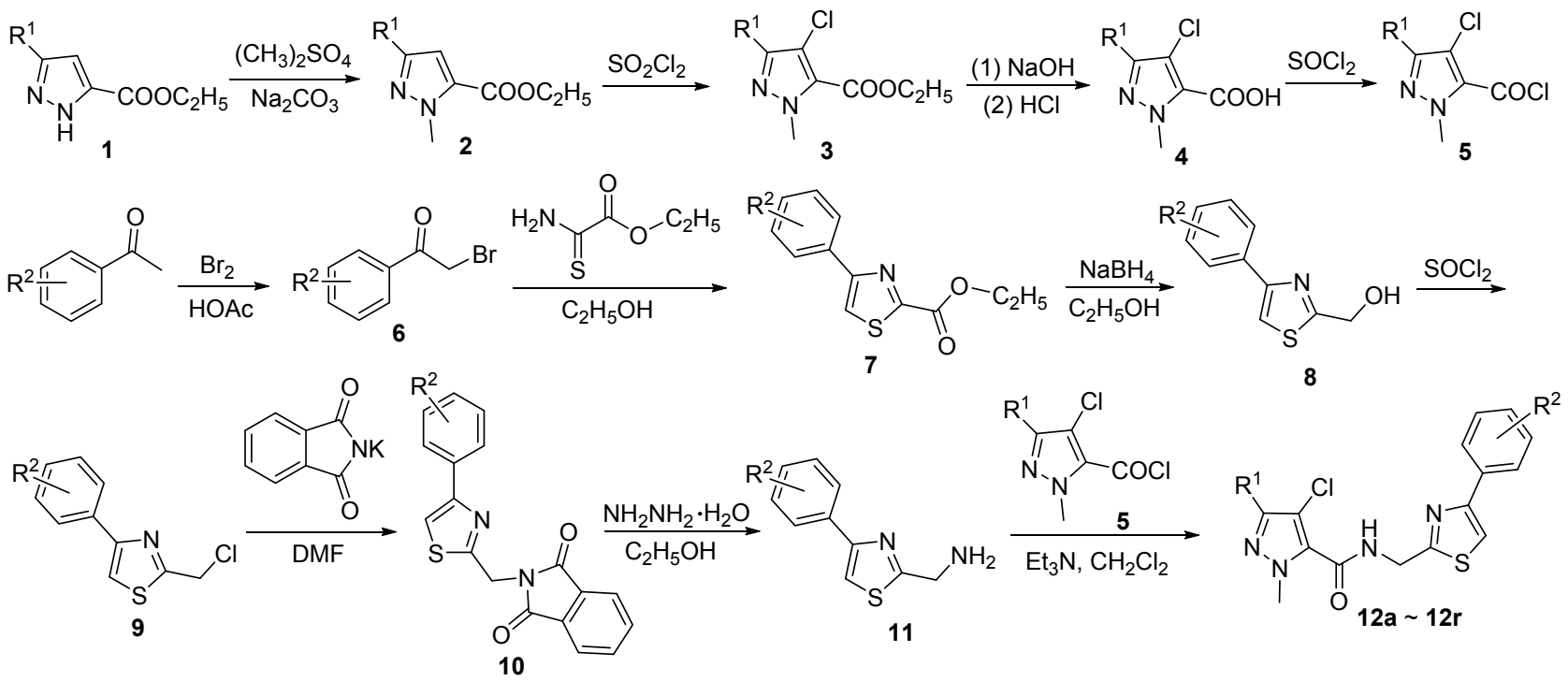

12a: $R^{1}=C_{3}, R^{2}=4-F ; 12 b: R^{1}=C_{3}, R^{2}=4-C l ; 12 c: R^{1}=C_{3}, R^{2}=4-B r ; 12 d: R^{1}=C_{3}, R^{2}=2,4-F_{2} ; 12 e: R^{1}=C_{3}, R^{2}=3,4-F_{2} ; 12 f:$ $R^{1}=C_{2} H_{5}, R^{2}=4-F ; 12 g: R^{1}=C_{2} H_{5}, R^{2}=4-C l ; 12 h: R^{1}=C_{2} H_{5}, R^{2}=4-B r ; 12 i: R^{1}=C_{2} H_{5}, R^{2}=2,4-F_{2} ; 12 j: R^{1}=C_{2} H_{5}, R^{2}=2,4-C l_{2} ; 12 k:$ $\mathrm{R}^{1}=\mathrm{C}_{2} \mathrm{H}_{5}, \mathrm{R}^{2}=3,4-\mathrm{F}_{2} ; 12 \mathrm{I}: \mathrm{R}^{1}=n-\mathrm{C}_{3} \mathrm{H}_{7}, \mathrm{R}^{2}=4-\mathrm{Cl} ; 12 \mathrm{~m}: \mathrm{R}^{1}=n-\mathrm{C}_{3} \mathrm{H}_{7}, \mathrm{R}^{2}=4-\mathrm{Br} ; 12 \mathrm{n}: \mathrm{R}^{1}=n-\mathrm{C}_{3} \mathrm{H}_{7}, \mathrm{R}^{2}=2,4-\mathrm{F}_{2} ; 12 \mathrm{o}: \mathrm{R}^{1}=n-\mathrm{C}_{3} \mathrm{H}_{7}, \mathrm{R}^{2}=$ $2,4-\mathrm{Cl}_{2} ; 12 p: \mathrm{R}^{1}=4-\mathrm{FC}_{6} \mathrm{H}_{4}, \mathrm{R}^{2}=2,4-\mathrm{F}_{2} ; 12 \mathrm{q}: \mathrm{R}^{1}=4-\mathrm{OCH}_{3} \mathrm{C}_{6} \mathrm{H}_{4}, \mathrm{R}^{2}=3,4-\mathrm{F}_{2} ; 12 \mathrm{r}: \mathrm{R}^{1}=2,4-\mathrm{Cl}_{2} \mathrm{C}_{6} \mathrm{H}_{3}, \mathrm{R}^{2}=2,4-\mathrm{Cl}_{2}$

图式 1 目标化合物 12 的合成路线

Scheme 1 Synthetic routes of the title compounds 12

表 1 不同反应条件对中间体 8 合成收率的影响

Table 1 Effects of reaction conditions on the synthesis of the intermediate 8

\begin{tabular}{ccccc}
\hline Entry & Reductant & $\mathbf{7}:$ Reductant & Solvent & Yield/\% \\
\hline 1 & $\mathrm{NaBH}_{4}$ & $1: 1$ & EtOH & 52 \\
2 & $\mathrm{NaBH}_{4}$ & $1: 2$ & EtOH & 63 \\
3 & $\mathrm{NaBH}_{4}$ & $1: 3$ & EtOH & 65 \\
4 & $\mathrm{NaBH}_{4}$ & $1: 4$ & EtOH & 78 \\
5 & $\mathrm{NaBH}_{4}$ & $1: 4$ & THF & 70 \\
6 & $\mathrm{LiAlH}_{4}$ & $1: 1$ & THF & 49 \\
7 & $\mathrm{LiAlH}_{4}$ & $1: 2$ & THF & 55 \\
8 & $\mathrm{LiAlH}_{4}$ & $1: 3$ & THF & 57 \\
9 & $\mathrm{LiAlH}_{4}$ & $1: 4$ & THF & 61 \\
10 & $\mathrm{LiAlH}_{4}$ & $1: 4$ & Et & 58 \\
\hline
\end{tabular}

1.2 化合物的谱图解析

以化合物 $12 \mathrm{~h}$ 为例, 对其 ${ }^{1} \mathrm{H}$ NMR 和 ${ }^{13} \mathrm{C}$ NMR 数据 进行解析. 从化合物 $\mathbf{1 2 h}$ 的 ${ }^{1} \mathrm{H}$ NMR 谱图可看出, $\mathrm{CONH}$ 上氢在 $\delta 7.69$ 以单峰出现, 噻唑环上的氢在 $\delta 7.46$ 以单 峰形式出现, 4-Br 取代苯环上四个氢在 $\delta 7.77$ 和 7.55 分 别以双重峰出现, 与 $\mathrm{CONH}$ 相连接的 $\mathrm{CH}_{2}$ 两个氢在 $\delta$ 4.98 以单峰形式出现，吡唑环 $N$-甲基上三个氢在 $\delta 4.16$ 以单峰形式出现, 吡唑环 3-位乙基上 $\mathrm{CH}_{2}$ 两个氢在 $\delta$ 2.67 以四重峰形式出现, 吡唑环 3 -位乙基上 $\mathrm{CH}_{3}$ 三个氢 在 $\delta 1.26$ 以三重峰形式出现; 从化合物 $\mathbf{1 2 h}$ 的 ${ }^{13} \mathrm{C} \mathrm{NMR}$ 数据数据可知, 酰胺键羰基碳原子的 $\delta$ 值为 158.6, 与 $\mathrm{CONH}$ 相连接的 $\mathrm{CH}_{2}$ 碳原子的 $\delta$ 值为 41.2, 吡唑环 $N-$ 甲基碳原子的 $\delta$ 值为 40.8 吡唑环 3-位乙基上 $\mathrm{CH}_{2}$ 碳
表 2 不同反应条件对目标化合物 $\mathbf{1 2 b}$ 合成收率的影响 Table 2 Effects of reaction conditions on the synthesis of the target compound 12b

\begin{tabular}{|c|c|c|c|c|}
\hline Entry & Base & $11: 5$ & Solvent & Yield/\% \\
\hline 1 & $\mathrm{Et}_{3} \mathrm{~N}$ & $1: 1$ & $\mathrm{CH}_{2} \mathrm{Cl}_{2}$ & 38 \\
\hline 2 & $\mathrm{Et}_{3} \mathrm{~N}$ & $1: 1.1$ & $\mathrm{CH}_{2} \mathrm{Cl}_{2}$ & 47 \\
\hline 3 & $\mathrm{Et}_{3} \mathrm{~N}$ & $1: 1.2$ & $\mathrm{CH}_{2} \mathrm{Cl}_{2}$ & 54 \\
\hline 4 & $\mathrm{Et}_{3} \mathrm{~N}$ & $1: 1.3$ & $\mathrm{CH}_{2} \mathrm{Cl}_{2}$ & 52 \\
\hline 5 & $\mathrm{Et}_{3} \mathrm{~N}$ & $1: 1.4$ & $\mathrm{CH}_{2} \mathrm{Cl}_{2}$ & 51 \\
\hline 6 & $\mathrm{Et}_{3} \mathrm{~N}$ & $1: 1.2$ & THF & 45 \\
\hline 7 & $\mathrm{Et}_{3} \mathrm{~N}$ & $1: 1.2$ & $\mathrm{CH}_{3} \mathrm{CN}$ & 40 \\
\hline 8 & Pyridine & $1: 1$ & $\mathrm{CH}_{2} \mathrm{Cl}_{2}$ & 32 \\
\hline 9 & Pyridine & $1: 1.1$ & $\mathrm{CH}_{2} \mathrm{Cl}_{2}$ & 38 \\
\hline 10 & Pyridine & $1: 1.2$ & $\mathrm{CH}_{2} \mathrm{Cl}_{2}$ & 46 \\
\hline 11 & Pyridine & $1: 1.3$ & $\mathrm{CH}_{2} \mathrm{Cl}_{2}$ & 43 \\
\hline 12 & Pyridine & $1: 1.4$ & $\mathrm{CH}_{2} \mathrm{Cl}_{2}$ & 41 \\
\hline 13 & Pyridine & $1: 1.2$ & THF & 39 \\
\hline 14 & Pyridine & $1: 1.2$ & $\mathrm{CH}_{3} \mathrm{CN}$ & 33 \\
\hline 15 & $\mathrm{~K}_{2} \mathrm{CO}_{3}$ & $1: 1.2$ & $\mathrm{CH}_{2} \mathrm{Cl}_{2}$ & 36 \\
\hline 16 & $\mathrm{~K}_{2} \mathrm{CO}_{3}$ & $1: 1.2$ & THF & 32 \\
\hline 17 & $\mathrm{~K}_{2} \mathrm{CO}_{3}$ & $1: 1.2$ & $\mathrm{CH}_{3} \mathrm{CN}$ & 30 \\
\hline
\end{tabular}

原子的 $\delta$ 值为 19.2, 吡唑环 3-位乙基上 $\mathrm{CH}_{3}$ 碳原子的 $\delta$ 值为 12.8 .

\section{3 杀虫活性}

通过浸叶法 ${ }^{[36]}$ 测定了目标化合物 $12 \mathrm{a} \sim 12 \mathrm{r}$ 对粘虫 (Oriental armyworm)的杀虫活性，另外采用喷雾法 ${ }^{[37}$ 测 定了目标化合物 12a 12r 对蚜虫(Aphis medicaginis)与 朱砂叶螨(Tetranychus cinnabarinus)的杀虫杀螨活性. 从 表 3 可以看出，化合物 $\mathbf{1 2 a} \sim \mathbf{1 2 r}$ 在 $500 \mu \mathrm{g} / \mathrm{mL}$ 浓度下对 
粘虫均有良好的杀虫效果, 其杀死率均为 $100 \%$, 明显 高于对照药剂吡螨胺的防效. 通过构效关系分析看出, 当 $\mathrm{R}^{1}=\mathrm{CH}_{3}$ 时, $\mathrm{R}^{2}$ 为 $4-\mathrm{Br}$ 的取代物 $12 \mathrm{c}$ 在 $100 \mu \mathrm{g} / \mathrm{mL}$ 时 对粘虫表现出中等水平的杀灭效果, 其杀虫活性为 $40 \%$, 要高于对照药剂吡螨胺的防效, 在其浓度降到 20 $\mu \mathrm{g} / \mathrm{mL}$ 时, 化合物 $12 \mathrm{c}$ 对粘虫仍有 $30 \%$ 的杀灭效果. 当 $\mathrm{R}^{1}=n-\mathrm{C}_{3} \mathrm{H}_{7}$ 时, $\mathrm{R}^{2}$ 为 $2,4-\mathrm{F}_{2}$ 取代物 $12 \mathrm{n}$ 在 $100 \mu \mathrm{g} / \mathrm{mL}$ 时 对粘虫的杀死率为 $30 \%$, 优于对照药剂吡螨胺的防效, 化合物 12n 在 $20 \mu \mathrm{g} / \mathrm{mL}$ 浓度下对粘虫仍有 $20 \%$ 的杀虫 活性. 部分目标化合物在 $500 \mu \mathrm{g} / \mathrm{mL}$ 时对蚜虫显示出优 良的杀虫效果. 当 $\mathrm{R}^{1}=\mathrm{CH}_{3}$ 时, $\mathrm{R}^{2}$ 为 $4-\mathrm{Br}$ 取代物 $12 \mathrm{c}$ 对 蚜虫的杀死率为 $100 \%$; 当 $\mathrm{R}^{1}=\mathrm{C}_{2} \mathrm{H}_{5}$ 时, $\mathrm{R}^{2}$ 为 $4-\mathrm{F}$ 取代 物 12f、 $R^{2}$ 为 4-Cl 取代物 $12 \mathrm{~g} 、 \mathrm{R}^{2}$ 为 $2,4-\mathrm{Cl}_{2}$ 取代物 $\mathbf{1 2} \mathbf{j}$ 对蚜虫的杀灭效果分别为 $70 \%, 100 \%$ 和 $80 \%$; 当 $\mathrm{R}^{1}=$ $n-\mathrm{C}_{3} \mathrm{H}_{7}$ 时, $\mathrm{R}^{2}$ 为 $4-\mathrm{Br}$ 取代物 $\mathbf{1 2 m}$ 和 $2,4-\mathrm{F}_{2}$ 取代物 $\mathbf{1 2 n}$ 对蚜虫的防效分别为 $80 \%$ 和 $100 \%$; 化合物 $12 \mathrm{p}\left(\mathrm{R}^{1}=\right.$ $4-\mathrm{FC}_{6} \mathrm{H}_{4}, \mathrm{R}^{2}=2,4-\mathrm{F}_{2}$ ) 对蚜虫也表现出 $70 \%$ 的杀虫活性. 化合物 12c、12m 和 12n 在 $100 \mu \mathrm{g} / \mathrm{mL}$ 浓度下对蚜虫具 有一定的杀虫作用，其杀死率分别 $20 \%, 30 \%$ 和 30\%. 另 外, 目标化合物 12c、12j 和 12n 在 $500 \mu \mathrm{g} / \mathrm{mL}$ 浓度下对 朱砂叶螨呈现出中等水平的杀虫效果, 杀死率分别为 $50 \%, 40 \%$ 和 50\%, 要低于对照药剂吡螨胺的药效. 这为 今后继续从事吡唑酰胺类化合物的研究提供了重要的 实验数据.

\section{2 结论}

基于杀螨剂吡螨胺的结构，制备出了 18 个含取代 噻唑单元的新型吡唑酰胺衍生物, 生物活性测定表明, 目标化合物 12a 12r 在 $500 \mu \mathrm{g} / \mathrm{mL}$ 浓度下对粘虫都有 $100 \%$ 的杀虫活性，均优于对照药剂吡螨胺的杀虫效果. 化合物 12c 和 12n 在 $100 \mu \mathrm{g} / \mathrm{mL}$ 时对粘虫表现出一定的 杀虫活性，其防效分别为 $40 \%$ 和 $30 \%$; 化合物 12c， 12f， $12 \mathrm{~g}, 12 \mathrm{j}, 12 \mathrm{~m}, 12 \mathrm{n}$ 和 12p 在 $500 \mu \mathrm{g} / \mathrm{mL}$ 时对蚜虫显示出 较好的防效，杀虫活性分别为 $100 \%, 70 \%, 100 \%, 80 \%$, $80 \%, 100 \%$ 和 $70 \%$; 此外，化合物 $\mathbf{1 2 c}, \mathbf{1 2} \mathbf{j}$ 和 $\mathbf{1 2 n}$ 在 500 $\mu \mathrm{g} / \mathrm{mL}$ 浓度时对朱砂叶螨具有 $50 \%, 40 \%$ 和 $50 \%$ 的杀灭 效果. 从总的构效关系分析也可以看出，目标化合物 12c $\left(\mathrm{R}^{1}=\mathrm{CH}_{3}, \mathrm{R}^{2}=4-\mathrm{Br}\right) 、 \mathbf{1 2 j}\left(\mathrm{R}^{1}=\mathrm{C}_{2} \mathrm{H}_{5}, \mathrm{R}^{2}=2,4-\mathrm{Cl}_{2}\right)$ 和 $12 \mathrm{n}\left(\mathrm{R}^{1}=n-\mathrm{C}_{3} \mathrm{H}_{7}, \mathrm{R}^{2}=2,4-\mathrm{F}_{2}\right)$ 对所测试的粘虫、蚜虫 和螨虫表现出较为广谱的杀虫作用，可作深入的结构优 化与杀虫活性探究.

\section{3 实验部分}

\section{1 仪器与试剂}

化合物的核磁氢谱、碳谱数据通过 BRUKER 400 $\mathrm{MHz}$ 核磁共振仪(TMS 为内标)测试; 化合物的熔点通过

表 3 目标化合物 12a $\sim 12 \mathrm{r}$ 的杀虫活性(死亡率/\%) $)^{a}$

Table 3 Insecticidal activities of compounds 12a $\sim 12 \mathbf{r}$ (mortality/\%)

\begin{tabular}{|c|c|c|c|c|c|c|c|c|}
\hline \multirow{2}{*}{ Compd. } & \multicolumn{3}{|c|}{ Oriental armyworm } & \multicolumn{3}{|c|}{ Aphis medicagini } & \multicolumn{2}{|c|}{ Tetranychus cinnabarinus } \\
\hline & $500 \mu \mathrm{g} \bullet \mathrm{mL}^{-1}$ & $100 \mu \mathrm{g} \cdot \mathrm{mL}^{-1}$ & $20 \mu \mathrm{g} \cdot \mathrm{mL}^{-1}$ & $500 \mu \mathrm{g} \cdot \mathrm{mL}^{-1}$ & $100 \mu \mathrm{g} \bullet \mathrm{mL}^{-1}$ & $20 \mu \mathrm{g} \cdot \mathrm{mL}^{-1}$ & $500 \mu \mathrm{g} \bullet \mathrm{mL}^{-1}$ & $100 \mu \mathrm{g} \cdot \mathrm{mL}^{-1}$ \\
\hline $12 a$ & 100 & 0 & - & 0 & - & - & 0 & - \\
\hline $12 b$ & 100 & 0 & - & 0 & - & - & 0 & - \\
\hline $12 \mathrm{c}$ & 100 & 40 & 30 & 100 & 20 & 0 & 50 & 0 \\
\hline 12d & 100 & 0 & - & 0 & - & - & 0 & - \\
\hline $12 \mathrm{e}$ & 100 & 0 & - & 0 & - & - & 0 & - \\
\hline $12 \mathrm{f}$ & 100 & 0 & - & 70 & 0 & - & 0 & - \\
\hline $12 \mathrm{~g}$ & 100 & 0 & - & 100 & 0 & - & 0 & - \\
\hline $12 \mathrm{~h}$ & 100 & 0 & - & 0 & - & - & 0 & - \\
\hline $12 \mathrm{i}$ & 100 & 0 & - & 0 & - & - & 0 & - \\
\hline $12 j$ & 100 & 0 & - & 80 & 0 & - & 40 & 0 \\
\hline $12 k$ & 100 & 0 & - & 0 & - & - & 0 & - \\
\hline 121 & 100 & 0 & - & 0 & - & - & 0 & - \\
\hline $12 \mathrm{~m}$ & 100 & 0 & - & 80 & 30 & 0 & 0 & - \\
\hline $12 n$ & 100 & 30 & 20 & 100 & 30 & 0 & 50 & 0 \\
\hline 120 & 100 & 0 & - & 0 & - & - & 0 & - \\
\hline $12 p$ & 100 & 0 & - & 70 & 0 & - & 0 & - \\
\hline $12 q$ & 100 & 0 & - & 0 & - & - & 0 & - \\
\hline $12 r$ & 100 & 0 & - & 0 & - & - & 0 & - \\
\hline Tolfenpyrad & 100 & 50 & 40 & - & - & - & - & - \\
\hline Imidacloprid & - & - & - & 100 & 100 & 100 & - & - \\
\hline Tebufenpyrad & 50 & 0 & - & - & - & - & 100 & 100 \\
\hline
\end{tabular}

"__" refers to not tested. 
X-4 显微熔点测定仪(温度计未经校正)测定; 化合 物的质谱数据经 Waters SQD-2 型质谱仪测试; 化合物 的元素分析数据经 Yanaco-CHN CORDER MT-3 自动元 素分析仪测试. 所用试剂都为分析纯.

\section{2 化合物 $1 \sim 5$ 和 6 的合成}

化合物 $1 \sim 5$ 的合成参照文献[38], 化合物 $\mathbf{6}$ 的合成 参照文献[39].

\section{3 化合物 7 的合成}

将中间体 6 (30 mmol)、硫代草氨酸乙酯 $(55 \mathrm{mmol})$ 及 $80 \mathrm{~mL}$ 无水乙醇加入一反应瓶中, 加热回流反应 6 $10 \mathrm{~h}$, 薄层色谱(TLC)跟踪反应的进程, 反应完成后, 将 反应液浓缩至干, 向其中加入 $50 \mathrm{~mL}$ 水, 用乙酸乙酯萃 取 $(60 \mathrm{~mL} \times 4)$, 有机相经无水硫酸镁干燥后, 抽滤浓缩 得到中间体 7, 化合物 7 可直接用于下面的反应. 为验 证化合物 7 的结构, 以取代基 $\mathrm{R}^{2}$ 为 4- $\mathrm{Cl}$ 的化合物为代 表, 通过乙醇重结晶得黄色固体 7, 产率 70\%. m.p. 99 $101{ }^{\circ} \mathrm{C} ;{ }^{1} \mathrm{H}$ NMR $\left(\mathrm{CDCl}_{3}, 400 \mathrm{MHz}\right) \delta: 1.46(\mathrm{t}, J=8.42$ $\mathrm{Hz}, 3 \mathrm{H}), 4.51$ (q, $J=8.40 \mathrm{~Hz}, 2 \mathrm{H}), 7.41(\mathrm{~d}, J=8.42 \mathrm{~Hz}$, $2 \mathrm{H}), 7.73(\mathrm{~s}, 1 \mathrm{H}), 7.91(\mathrm{~d}, J=8.40 \mathrm{~Hz}, 2 \mathrm{H})$. Anal. calcd for $\mathrm{C}_{12} \mathrm{H}_{10} \mathrm{ClNO}_{2} \mathrm{~S}$ : C 53.84, H 3.77, N 5.23; found C 53.95, H 3.93, N 5.09 .

\section{4 化合物 8 的合成}

将中间体 $7(18 \mathrm{mmol})$ 及 $100 \mathrm{~mL}$ 无水乙醇加入一反 应瓶中, 冰浴搅拌下, 向其中缓慢加入硼氢化钠 (72 $\mathrm{mmol}$ ), 加完后, 继续冰浴搅拌 $3 \sim 6 \mathrm{~h}$, 反应完成后, 向 反应液中加入 $20 \mathrm{~mL}$ 水, 用稀盐酸调节 $\mathrm{pH}$ 至 7 , 静置析 出固体, 抽滤得到中间体 $\mathbf{8}$, 化合物 $\mathbf{8}$ 可直接用于后面 的反应. 为验证化合物 8 的结构, 以取代基 $\mathrm{R}^{2}$ 为 4-Cl 的化合物为代表, 经乙醇重结晶得白色固体 $\mathbf{8}$, 产率 78\%. m.p. $98 \sim 100{ }^{\circ} \mathrm{C} ;{ }^{1} \mathrm{H}$ NMR $\left(\mathrm{CDCl}_{3}, 400 \mathrm{MHz}\right) \delta$ : $5.00(\mathrm{~s}, 2 \mathrm{H}), 7.39$ (d, $J=8.81 \mathrm{~Hz}, 2 \mathrm{H}), 7.45(\mathrm{~s}, 1 \mathrm{H}), 7.82$ (d, $J=7.22 \mathrm{~Hz}, 2 \mathrm{H}$ ). Anal. calcd for $\mathrm{C}_{10} \mathrm{H}_{8} \mathrm{CINOS:} \mathrm{C}$ 53.22, H 3.57, N 6.21; found C 53.05, H 3.41, N 6.33.

\section{5 化合物 9 的合成}

将中间体 $8(12 \mathrm{mmol})$ 及 $30 \mathrm{~mL}$ DMF 加入反应瓶中, 冰浴搅拌下, 慢慢滴加二氯亚砜 (50 mmol), 加好后, 室 温反应 $2 \sim 6 \mathrm{~h}$, 反应结束后, 将反应液浓缩至干, 向反 应瓶中加入 $20 \mathrm{~mL}$ 水, 用饱和 $\mathrm{NaHCO}_{3}$ 调节 $\mathrm{pH}$ 至 8 , 静 置析出固体, 经抽滤、烘干得中间体 $\mathbf{9}$, 不经纯化可直接 用于下一步反应.

\section{6 化合物 11 的合成}

将中间体 9 (8 mmol)、邻苯二甲酰亚胺钾盐(16 $\mathrm{mmol}$ )及 $50 \mathrm{~mL} N, N$-二甲基甲酰胺(DMF)加入一反应瓶
中, 加完后, 室温反应 2 5 h, TLC 跟进反应的进程. 反 应结束后, 将反应液倒入盛有 $200 \mathrm{~mL}$ 水的烧杯中, 静 置析出固体, 经抽滤、烘干得中间体 $\mathbf{1 0}$, 不经纯化可用 于后面的反应. 将前面所得中间体 $\mathbf{1 0}(6 \mathrm{mmol}) 、 80 \%$ 水 合肼 $(18 \mathrm{mmol})$ 及 $30 \mathrm{~mL}$ 无水乙醇加入一反应瓶中, 加 完后, 加热回流反应 $3 \sim 6 \mathrm{~h}$. 反应完成后, 经过滤、浓缩 得到中间体 11, 可不经提纯化直接投入下一步反应.

\subsection{4 -氯-1,3-二取代基- $N$-[(4-取代苯基噻唑-2-基)甲 基]-1 $H$-吡唑-5-甲酰胺(12a 12r)的合成}

将中间体 $11(4 \mathrm{mmol}) 、$ 三胺 $(5 \mathrm{mmol})$ 和 $25 \mathrm{~mL}$ 二 氯甲烷加入一反应瓶中, 冰浴搅拌下, 向其中缓慢滴加 $5 \mathrm{mmol}$ 中间体 5 的二氯甲烷 $(5 \mathrm{~mL})$ 溶液. 滴加完后, 室 温反应 6 12 h, TLC 监测反应进程. 完全结束后, 经过 滤、浓缩所得粗品经柱层析 (以乙酸乙酯/石油醚为洗脱 剂, $V: V=1: 2$ )分离得到目标化合物 $\mathbf{1 2 a} \sim 12 \mathbf{r}$.

4-氯-1,3-二甲基- $N$-\{[4-(4-氟苯基)噻唑-2-基]甲 基\}-1 $H$-吡唑-5-甲酰胺(12a): 白色固体, 产率 54\%. m.p. $160 \sim 162{ }^{\circ} \mathrm{C} ;{ }^{1} \mathrm{H}$ NMR $\left(\mathrm{CDCl}_{3}, 400 \mathrm{MHz}\right) \delta: 7.86 \sim 7.89$ (m, 2H), $7.72(\mathrm{~s}, 1 \mathrm{H}), 7.40(\mathrm{~s}, 1 \mathrm{H}), 7.12(\mathrm{t}, J=8.80 \mathrm{~Hz}$, 2H), 4.99 (d, $J=5.60 \mathrm{~Hz}, 2 \mathrm{H}), 4.15$ (s, 3H), 2.26 (s, 3H); ${ }^{13} \mathrm{C} \mathrm{NMR}\left(100 \mathrm{MHz}, \mathrm{CDCl}_{3}\right) \delta: 166.3,164.0,161.6,158.5$, $154.2,144.7,130.5,128.1,128.0,115.9,115.7,112.9$, 108.9, 41.2, 40.7, 11.1; ESI-MS $m / z: 365[\mathrm{M}+\mathrm{H}]^{+}$. Anal. calcd for $\mathrm{C}_{16} \mathrm{H}_{14} \mathrm{ClFN}_{4} \mathrm{OS}$ : C 52.67, H 3.87, N 15.36; found C 52.83, H 3.99, N 15.21.

4-氯-1,3-二甲基- $N$ - $\{[4-$-(4-氯苯基)噻唑-2-基]甲 基 \}-1H-吡唑-5-甲酰胺(12b): 黄色固体, 产率 54\%. m.p. 147 $149{ }^{\circ} \mathrm{C} ;{ }^{1} \mathrm{H}$ NMR $\left(\mathrm{CDCl}_{3}, 400 \mathrm{MHz}\right) \delta: 7.83$ (d, $J=$ $8.40 \mathrm{~Hz}, 2 \mathrm{H}), 7.69$ (s, 1H), 7.45 (s, 1H), 7.40 (d, $J=8.40$ $\mathrm{Hz}, 2 \mathrm{H}), 4.99$ (d, J=5.60 Hz, 2H), 4.15 (s, 3H), 2.26 (s, $3 \mathrm{H}) ;{ }^{13} \mathrm{C}$ NMR $\left(100 \mathrm{MHz}, \mathrm{CDCl}_{3}\right) \delta: 166.4,158.5,154.0$, $144.7,134.2,132.5,130.5,129.0,127.6,113.6,109.0$, 41.2, 40.8, 11.1; ESI-MS $m / z: 381[\mathrm{M}+\mathrm{H}]^{+}$. Anal. calcd for $\mathrm{C}_{16} \mathrm{H}_{14} \mathrm{Cl}_{2} \mathrm{~N}_{4} \mathrm{OS}$ : C 50.40, H 3.70, N 14.69; found $\mathrm{C}$ $50.27, \mathrm{H} 3.86, \mathrm{~N} 14.84$.

4-氯-1,3-二甲基- $N$-\{[4-(4-溴苯基)噻唑-2-基]甲 基\}-1H-吡唑-5-甲酰胺(12c): 黄色固体, 产率 52\%. m.p. $161 \sim 163{ }^{\circ} \mathrm{C} ;{ }^{1} \mathrm{H}$ NMR $\left(\mathrm{CDCl}_{3}, 400 \mathrm{MHz}\right) \delta: 7.56(\mathrm{~d}$, $J=8.80 \mathrm{~Hz}, 2 \mathrm{H}), 7.49$ (d, $J=8.40 \mathrm{~Hz}, 2 \mathrm{H}), 7.46(\mathrm{~s}, 1 \mathrm{H})$, $7.31(\mathrm{~s}, 1 \mathrm{H}), 4.82(\mathrm{~d}, J=5.20 \mathrm{~Hz}, 2 \mathrm{H}), 4.14(\mathrm{~s}, 3 \mathrm{H}), 2.25$ (s, 3H); ${ }^{13} \mathrm{C}$ NMR $\left(100 \mathrm{MHz}, \mathrm{CDCl}_{3}\right) \delta: 166.4,158.5$, $154.1,144.7,132.9,132.0,130.5,127.9,122.4,113.7$, 109.0, 41.2, 40.8, 11.1; ESI-MS $m / z: 425[\mathrm{M}+\mathrm{H}]^{+}$. Anal. calcd for $\mathrm{C}_{16} \mathrm{H}_{14} \mathrm{BrClN}_{4} \mathrm{OS}$ : C 45.14, H 3.31, N 13.16; found C 44.98, H 3.43, N 13.01. 
4-氯-1,3-二甲基- $N$ - $\{[4-(2,4-$ 二氟苯基)噻唑-2-基]甲 基\}-1H-吡唑-5-甲酰胺(12d)：淡黄色固体，产率 50\%. m.p. $164 \sim 166{ }^{\circ} \mathrm{C} ;{ }^{1} \mathrm{H}$ NMR $\left(\mathrm{CDCl}_{3}, 400 \mathrm{MHz}\right) \delta: 8.16 \sim$ $8.23(\mathrm{~m}, 1 \mathrm{H}), 7.67(\mathrm{~d}, J=2.40 \mathrm{~Hz}, 2 \mathrm{H}), 6.89 \sim 7.00(\mathrm{~m}$, 2H), 4.99 (d, J=5.20 Hz, 2H), 4.16 (s, 3H), 2.26 (s, 3H); ${ }^{13} \mathrm{C}$ NMR $\left(100 \mathrm{MHz}, \mathrm{CDCl}_{3}\right) \delta: 165.3,163.7,161.4,159.0$, $147.9,144.7,130.9,130.5,117.5,111.9,108.9,104.4$, 41.1, 40.8, 11.1; ESI-MS m/z: $383[\mathrm{M}+\mathrm{H}]^{+}$. Anal. calcd for $\mathrm{C}_{16} \mathrm{H}_{13} \mathrm{ClF}_{2} \mathrm{~N}_{4} \mathrm{OS}$ : C 50.20, $\mathrm{H} 3.42, \mathrm{~N} 14.64$; found $\mathrm{C}$ 50.06, H 3.25, N 14.79.

4-氯-1,3-二甲基- $N$ - $\{[4$-(3,4-二氟苯基)噻唑-2-基]甲 基\}-1H-吡唑-5-甲酰胺(12e)：淡黄色固体，产率 53\%. m.p. $173 \sim 175{ }^{\circ} \mathrm{C} ;{ }^{1} \mathrm{H}$ NMR $\left(\mathrm{CDCl}_{3}, 400 \mathrm{MHz}\right) \delta: 7.58 \sim$ $7.75(\mathrm{~m}, 3 \mathrm{H}), 7.42(\mathrm{~s}, 1 \mathrm{H}), 7.18 \sim 7.24(\mathrm{~m}, 1 \mathrm{H}), 4.97$ (d, $J=5.60 \mathrm{~Hz}, 2 \mathrm{H}), 4.15$ (s, 3H), 2.27 (s, 3H); ${ }^{13} \mathrm{C} \mathrm{NMR}(100$ $\left.\mathrm{MHz}, \mathrm{CDCl}_{3}\right) \delta: 166.6,158.5,153.1,151.8,149.3,144.7$, 131.3, 130.5, 122.2, 117.7, 115.5, 113.8, 109.0, 41.2, 40.8, 11.1; ESI-MS $m / z$ : $383[\mathrm{M}+\mathrm{H}]^{+}$. Anal. calcd for $\mathrm{C}_{16} \mathrm{H}_{13} \mathrm{ClF}_{2} \mathrm{~N}_{4} \mathrm{OS}$ : C 50.20, $\mathrm{H}$ 3.42, $\mathrm{N}$ 14.64; found $\mathrm{C}$ 50.33, H 3.58, N 14.50 .

4-氯-1-甲基-3-乙基- $N$ - $\{$ [4-(4-氟苯基)噻唑-2-基]甲 基\}-1H-吡唑-5-甲酰胺(12f)：白色固体，产率 51\%. m.p. $115 \sim 117{ }^{\circ} \mathrm{C} ;{ }^{1} \mathrm{H}$ NMR $\left(\mathrm{CDCl}_{3}, 400 \mathrm{MHz}\right) \delta: 7.59 \sim 7.62$ $(\mathrm{m}, 2 \mathrm{H}), 7.47(\mathrm{~s}, 1 \mathrm{H}), 7.24(\mathrm{~s}, 1 \mathrm{H}), 7.12(\mathrm{t}, J=8.40 \mathrm{~Hz}$, $2 \mathrm{H}), 4.82(\mathrm{~d}, J=5.20 \mathrm{~Hz}, 2 \mathrm{H}), 4.15(\mathrm{~s}, 3 \mathrm{H}), 2.65$ (q, $J=7.60 \mathrm{~Hz}, 2 \mathrm{H}), 1.25(\mathrm{t}, J=7.60 \mathrm{~Hz}, 3 \mathrm{H}) ;{ }^{13} \mathrm{C}$ NMR $(100$ $\left.\mathrm{MHz}, \mathrm{CDCl}_{3}\right) \delta: 164.0,161.6,159.5,158.6,151.4,149.7$, $130.5,126.2$, 123.9, 121.5, 116.3, 116.0, 108.2, 40.8, 39.9, 19.2, 12.8; ESI-MS m/z: $379[\mathrm{M}+\mathrm{H}]^{+}$. Anal. calcd for $\mathrm{C}_{17} \mathrm{H}_{16} \mathrm{ClFN}_{4} \mathrm{OS}$ : C 53.89, H 4.26, N 14.79; found C 53.72, $\mathrm{H} 4.15, \mathrm{~N} 14.94$.

4-氯-1-甲基-3-乙基- $N$ - $\{$ [4-(4-氯苯基)噻唑-2-基]甲 基\}-1H-吡唑-5-甲酰胺(12g)：白色固体，产率 55\%. m.p. $149 \sim 151{ }^{\circ} \mathrm{C}$; ${ }^{1} \mathrm{H}$ NMR $\left(\mathrm{CDCl}_{3}, 400 \mathrm{MHz}\right) \delta: 7.83$ (d, $J=8.40 \mathrm{~Hz}, 2 \mathrm{H}), 7.69$ (s, 1H), 7.45 (s, 1H), 7.40 (d, $J=$ $8.80 \mathrm{~Hz}, 2 \mathrm{H}), 4.99$ (d, J=5.60 Hz, 2H), 4.16 (s, 3H), 2.67 (q, $J=7.60 \mathrm{~Hz}, 2 \mathrm{H}), 1.26(\mathrm{t}, J=7.60 \mathrm{~Hz}, 3 \mathrm{H}) ;{ }^{13} \mathrm{C} \mathrm{NMR}$ $\left(100 \mathrm{MHz}, \mathrm{CDCl}_{3}\right) \delta: 166.5,158.6,153.9,149.7,134.2$, 132.4, 130.5, 129.0, 127.6, 113.7, 108.2, 41.1, 40.8, 19.2, 12.8; ESI-MS $m / z$ : $395[\mathrm{M}+\mathrm{H}]^{+}$. Anal. calcd for $\mathrm{C}_{17} \mathrm{H}_{16} \mathrm{Cl}_{2} \mathrm{~N}_{4} \mathrm{OS}$ : C 51.65, H 4.08, N 14.17; found C 51.81, H 3.93, N 14.04.

4-氯-1-甲基-3-乙基- $N$ - $\{$ [4-(4-溴苯基)噻唑-2-基]甲 基\}-1H-吡唑-5-甲酰胺(12h)：淡黄色固体，产率 49\%. m.p. $>280{ }^{\circ} \mathrm{C} ;{ }^{1} \mathrm{H} \mathrm{NMR}\left(\mathrm{CDCl}_{3}, 400 \mathrm{MHz}\right) \delta: 7.77$ (d,
$J=8.40 \mathrm{~Hz}, 2 \mathrm{H}), 7.69$ (s, 1H), 7.55 (d, $J=8.80 \mathrm{~Hz}, 2 \mathrm{H})$, $7.46(\mathrm{~s}, 1 \mathrm{H}), 4.98$ (d, $J=5.60 \mathrm{~Hz}, 2 \mathrm{H}), 4.16$ (s, 3H), 2.67 (q, $J=7.60 \mathrm{~Hz}, 2 \mathrm{H}), 1.26(\mathrm{t}, J=7.60 \mathrm{~Hz}, 3 \mathrm{H}) ;{ }^{13} \mathrm{C} \mathrm{NMR}$ $\left(100 \mathrm{MHz}, \mathrm{CDCl}_{3}\right) \delta: 166.4,158.6,154.1,149.7,133.0$, 132.0, 130.5, 127.8, 122.3, 113.7, 108.2, 41.2, 40.8, 19.2, 12.8; ESI-MS $m / z$ : $439[\mathrm{M}+\mathrm{H}]^{+}$. Anal. calcd for $\mathrm{C}_{17} \mathrm{H}_{16^{-}}$ $\mathrm{BrClN}_{4} \mathrm{OS}$ : C 46.43, H 3.67, N 12.74; found C 46.56, H $3.50, \mathrm{~N} 12.88$.

4-氯-1-甲基-3-乙基- $N$ - $\{[4-(2,4$-二氟苯基)噻唑-2基]甲基\}- $1 H$-吡唑-5-甲酰胺(12i)：白色固体，产率 53\%. m.p. $153 \sim 155{ }^{\circ} \mathrm{C} ;{ }^{1} \mathrm{H}$ NMR $\left(\mathrm{CDCl}_{3}, 400 \mathrm{MHz}\right) \delta: 8.17 \sim$ $8.23(\mathrm{~m}, 1 \mathrm{H}), 7.68(\mathrm{~d}, J=2.40 \mathrm{~Hz}, 2 \mathrm{H}), 6.88 \sim 7.01(\mathrm{~m}$, 2H), 4.99 (d, $J=5.60 \mathrm{~Hz}, 2 \mathrm{H}), 4.16(\mathrm{~s}, 3 \mathrm{H}), 2.67$ (q, $J=$ $7.60 \mathrm{~Hz}, 2 \mathrm{H}), 1.26(\mathrm{t}, J=7.60 \mathrm{~Hz}, 3 \mathrm{H}) ;{ }^{13} \mathrm{C}$ NMR $(100$ $\left.\mathrm{MHz}, \mathrm{CDCl}_{3}\right) \delta: 166.6,158.6,155.0,151.9,149.8,149.3$, $130.5,122.3,117.6,115.4,113.8,108.2,41.2,40.8,19.2$, 12.8; ESI-MS m/z: $397[\mathrm{M}+\mathrm{H}]^{+}$. Anal. calcd for $\mathrm{C}_{17} \mathrm{H}_{15} \mathrm{ClF}_{2} \mathrm{~N}_{4} \mathrm{OS}$ : C 51.45, $\mathrm{H}$ 3.81, N 14.12; found $\mathrm{C}$ 51.31, H 3.92, N 14.27 .

4-氯-1-甲基-3-乙基- $N$ - $\{[4-(2,4$-二氯苯基)噻唑-2基]甲基 $\}-1 H$-吡唑-5-甲酰胺(12j)：淡黄色固体，产率 48\%. m.p. 162 164 ${ }^{\circ} \mathrm{C} ;{ }^{1} \mathrm{H}$ NMR $\left(\mathrm{CDCl}_{3}, 400 \mathrm{MHz}\right) \delta$ : $7.93(\mathrm{~d}, J=8.40 \mathrm{~Hz}, 1 \mathrm{H}), 7.84$ (s, 1H), 7.67 (s, 1H), 7.50 $(\mathrm{d}, J=2.00 \mathrm{~Hz}, 1 \mathrm{H}), 7.33 \sim 7.36(\mathrm{~m}, 1 \mathrm{H}), 5.01(\mathrm{~d}, J=5.60$ $\mathrm{Hz}, 2 \mathrm{H}), 4.16$ (s, 3H), 2.66 (q, J=7.60 Hz, 2H), 1.25 (t, $J=7.60 \mathrm{~Hz}, 3 \mathrm{H}) ;{ }^{13} \mathrm{C} \mathrm{NMR}\left(100 \mathrm{MHz}, \mathrm{CDCl}_{3}\right) \delta: 165.3$, $158.6,150.4,149.7,134.3,132.5,132.2,131.3,130.5$, 130.3, 127.4, 118.9, 108.2, 41.1, 40.8, 19.2, 12.8; ESI-MS $m / z: 429[\mathrm{M}+\mathrm{H}]^{+}$. Anal. calcd for $\mathrm{C}_{17} \mathrm{H}_{15} \mathrm{Cl}_{3} \mathrm{~N}_{4} \mathrm{OS}: \mathrm{C}$ 47.51, H 3.52, N 13.04; found C 47.68, H 3.36, N 13.16.

4-氯-1-甲基-3-乙基- $N$ - $\{[4-(3,4$-二氟苯基)噻唑-2基]甲基 $\}-1 H$-吡唑-5-甲酰胺(12k)：白色固体，产率 49\%. m.p. $149 \sim 151{ }^{\circ} \mathrm{C} ;{ }^{1} \mathrm{H}$ NMR $\left(\mathrm{CDCl}_{3}, 400 \mathrm{MHz}\right) \delta$ : $7.68 \sim 7.75(\mathrm{~m}, 2 \mathrm{H}), 7.60 \sim 7.63(\mathrm{~m}, 1 \mathrm{H}), 7.42(\mathrm{~s}, 1 \mathrm{H})$, $7.18 \sim 7.24(\mathrm{~m}, 1 \mathrm{H}), 4.98(\mathrm{~d}, J=5.60 \mathrm{~Hz}, 2 \mathrm{H}), 4.16(\mathrm{~s}$, $3 \mathrm{H}), 2.67$ (q, $J=7.60 \mathrm{~Hz}, 2 \mathrm{H}), 1.25$ (t, $J=7.60 \mathrm{~Hz}, 3 \mathrm{H})$; ${ }^{13} \mathrm{C}$ NMR $\left(100 \mathrm{MHz}, \mathrm{CDCl}_{3}\right) \delta: 166.8,158.6,153.0,149.8$, $131.2,130.5,122.3,117.8,117.6,115.6,115.4,113.8$, 108.2, 41.2, 40.8, 19.3, 12.8; ESI-MS m/z: $397[\mathrm{M}+\mathrm{H}]^{+}$. Anal. calcd for $\mathrm{C}_{17} \mathrm{H}_{15} \mathrm{ClF}_{2} \mathrm{~N}_{4} \mathrm{OS}$ : C 51.45, H 3.81, N 14.12; found C 51.62, H 3.67, N 14.03.

4-氯-1-甲基-3-正丙基- $N$ - $\{$ [4-(4-氯苯基)噻唑-2-基] 甲基 $\}-1 H$-吡唑-5-甲酰胺(12l)：白色固体，产率 51\%. m.p. $>280{ }^{\circ} \mathrm{C} ;{ }^{1} \mathrm{H} \mathrm{NMR}\left(\mathrm{CDCl}_{3}, 400 \mathrm{MHz}\right) \delta: 7.83$ (d, $J=8.40 \mathrm{~Hz}, 2 \mathrm{H}), 7.69$ (s, 1H), 7.44 (s, 1H), 7.40 (d, $J=$ 
$8.40 \mathrm{~Hz}, 2 \mathrm{H}), 4.98(\mathrm{~d}, J=5.60 \mathrm{~Hz}, 2 \mathrm{H}), 4.16(\mathrm{~s}, 3 \mathrm{H}), 2.61$ $(\mathrm{t}, J=7.20 \mathrm{~Hz}, 2 \mathrm{H}), 1.66 \sim 1.72(\mathrm{~m}, 2 \mathrm{H}), 0.98(\mathrm{t}, J=7.20$ $\mathrm{Hz}, 3 \mathrm{H}) ;{ }^{13} \mathrm{C} \mathrm{NMR}\left(100 \mathrm{MHz}, \mathrm{CDCl}_{3}\right) \delta: 166.4,158.6$, $154.0,148.5,134.1,132.5,130.5,129.0,127.6,113.6$, 108.6, 41.2, 40.8, 27.7, 21.9, 13.8; ESI-MS m/z: 409 [M+ $\mathrm{H}]^{+}$. Anal. calcd for $\mathrm{C}_{18} \mathrm{H}_{18} \mathrm{Cl}_{2} \mathrm{~N}_{4} \mathrm{OS}$ : C 52.82, $\mathrm{H} 4.43, \mathrm{~N}$ 13.69; found $\mathrm{C} 52.68, \mathrm{H} 4.59, \mathrm{~N} 13.81$.

4-氯-1-甲基-3-正丙基- $N$ - $\{[4$-(4-溴苯基)噻唑-2-基 $]$ 甲基\}-1H-吡唑-5-甲酰胺(12m): 白色固体，产率 47\%. m.p. $122 \sim 124{ }^{\circ} \mathrm{C} ;{ }^{1} \mathrm{H}$ NMR $\left(\mathrm{CDCl}_{3}, 400 \mathrm{MHz}\right) \delta: 7.77$ $(\mathrm{d}, J=8.40 \mathrm{~Hz}, 2 \mathrm{H}), 7.70(\mathrm{~s}, 1 \mathrm{H}), 7.56(\mathrm{~d}, J=8.40 \mathrm{~Hz}$, 2H), 7.46 (s, 1H), 4.99 (d, J=5.60 Hz, 2H), 4.16 (s, 3H), $2.61(\mathrm{t}, J=7.60 \mathrm{~Hz}, 2 \mathrm{H}), 1.66 \sim 1.72(\mathrm{~m}, 2 \mathrm{H}), 0.98(\mathrm{t}, J=$ $7.20 \mathrm{~Hz}, 3 \mathrm{H}) ;{ }^{13} \mathrm{C} \mathrm{NMR}\left(100 \mathrm{MHz}, \mathrm{CDCl}_{3}\right) \delta: 166.6$, $158.6,153.9,148.6,132.8,132.0,130.4,127.9,122.4$, 113.8, 108.6, 41.1, 40.8, 27.7, 21.9, 13.8; ESI-MS m/z: 453 $[\mathrm{M}+\mathrm{H}]^{+}$. Anal. calcd for $\mathrm{C}_{18} \mathrm{H}_{18} \mathrm{BrClN}_{4} \mathrm{OS}$ : C 47.64, H 4.00, N 12.35; found C 47.50, H 4.16, N 12.22.

4-氯-1-甲基-3-正丙基- $N$ - $\{[4-(2,4$-二氟苯基)噻唑-2基]甲基 $\}-1 H$-吡唑-5-甲酰胺(12n)：白色固体，产率 50\%. m.p. $135 \sim 137{ }^{\circ} \mathrm{C} ;{ }^{1} \mathrm{H}$ NMR $\left(\mathrm{CDCl}_{3}, 400 \mathrm{MHz}\right) \delta$ : $8.16 \sim 8.22(\mathrm{~m}, 1 \mathrm{H}), 7.67(\mathrm{~d}, J=2.02 \mathrm{~Hz}, 2 \mathrm{H}), 6.88 \sim 7.00$ $(\mathrm{m}, 2 \mathrm{H}), 4.98(\mathrm{~d}, J=5.60 \mathrm{~Hz}, 2 \mathrm{H}), 4.16(\mathrm{~s}, 3 \mathrm{H}), 2.61(\mathrm{t}$, $J=7.60 \mathrm{~Hz}, 2 \mathrm{H}), 1.66 \sim 1.72(\mathrm{~m}, 2 \mathrm{H}), 0.98(\mathrm{t}, J=7.60 \mathrm{~Hz}$, $3 \mathrm{H}) ;{ }^{13} \mathrm{C} \mathrm{NMR}\left(100 \mathrm{MHz}, \mathrm{CDCl}_{3}\right) \delta: 165.3,163.6,161.4$, $158.6,148.5,147.9,131.0,130.5,118.5,117.5,111.9$, 108.5, 104.4, 41.2, 40.8, 27.7, 21.9, 13.8; ESI-MS m/z: 411 $[\mathrm{M}+\mathrm{H}]^{+}$. Anal. calcd for $\mathrm{C}_{18} \mathrm{H}_{17} \mathrm{ClF}_{2} \mathrm{~N}_{4} \mathrm{OS}$ : C 52.62, $\mathrm{H}$ 4.17, N 13.64; found C 52.47, H 4.33, N 13.76.

4-氯-1-甲基-3-正丙基- $N$ - $\{[4-(2,4$-二氯苯基)噻唑-2基]甲基\}-1H-吡唑-5-甲酰胺(120)：白色固体，产率 52\%. m.p. $120 \sim 122{ }^{\circ} \mathrm{C} ;{ }^{1} \mathrm{H}$ NMR $\left(\mathrm{CDCl}_{3}, 400 \mathrm{MHz}\right) \delta: 7.93$ (d, $J=8.40 \mathrm{~Hz}, 1 \mathrm{H}), 7.84(\mathrm{~s}, 1 \mathrm{H}), 7.66(\mathrm{~s}, 1 \mathrm{H}), 7.49$ (d, $J=2.00 \mathrm{~Hz}, 1 \mathrm{H}), 7.33 \sim 7.35(\mathrm{~m}, 1 \mathrm{H}), 4.99(\mathrm{~d}, J=5.20 \mathrm{~Hz}$, $2 \mathrm{H}), 4.16(\mathrm{~s}, 3 \mathrm{H}), 2.60(\mathrm{t}, J=7.20 \mathrm{~Hz}, 2 \mathrm{H}), 1.66 \sim 1.71(\mathrm{~m}$, $2 \mathrm{H}), 0.97(\mathrm{t}, J=7.60 \mathrm{~Hz}, 3 \mathrm{H}) ;{ }^{13} \mathrm{C} \mathrm{NMR}(100 \mathrm{MHz}$, $\left.\mathrm{CDCl}_{3}\right) \delta: 165.3,158.6,150.4,148.5,134.3,132.5,132.2$, 131.3, 130.4, 130.3, 127.4, 118.9, 108.6, 41.1, 40.8, 27.7, 21.9, 13.8; ESI-MS m/z: $443[\mathrm{M}+\mathrm{H}]^{+}$. Anal. calcd for $\mathrm{C}_{18} \mathrm{H}_{17} \mathrm{Cl}_{3} \mathrm{~N}_{4} \mathrm{OS}$ : C 48.72, H 3.86, N 12.62; found C 48.89, $\mathrm{H} 3.73, \mathrm{~N} 12.51$.

4-氯-1-甲基-3-(4-氟苯基)- $N$ - $\{[4-(2,4$-二氟苯基)噻 唑-2-基]甲基\}-1H-吡唑-5-甲酰胺(12p)：白色固体, 产率 48\%. m.p. $172 \sim 174{ }^{\circ} \mathrm{C} ;{ }^{1} \mathrm{H}$ NMR $\left(\mathrm{CDCl}_{3}, 400 \mathrm{MHz}\right) \delta$ : $8.16 \sim 8.22(\mathrm{~m}, 1 \mathrm{H}), 7.80 \sim 7.84(\mathrm{~m}, 2 \mathrm{H}), 7.78(\mathrm{~s}, 1 \mathrm{H})$, $7.69(\mathrm{~s}, 1 \mathrm{H}), 7.12 \sim 7.17(\mathrm{~m}, 2 \mathrm{H}), 6.89 \sim 7.01(\mathrm{~m}, 2 \mathrm{H})$, $5.02(\mathrm{~d}, J=5.20 \mathrm{~Hz}, 2 \mathrm{H}), 4.25(\mathrm{~s}, 3 \mathrm{H}) ;{ }^{13} \mathrm{C}$ NMR $(100$ $\left.\mathrm{MHz}, \mathrm{CDCl}_{3}\right) \delta: 165.0,164.2,163.6,161.7,159.0,158.4$, $147.9,145.6,131.8,130.9,129.5,126.9,117.5,115.7$, 115.5, 111.9, 111.7, 107.3, 104.4, 41.3, 41.3; ESI-MS m/z: $463[\mathrm{M}+\mathrm{H}]^{+}$. Anal. calcd for $\mathrm{C}_{21} \mathrm{H}_{14} \mathrm{ClF}_{3} \mathrm{~N}_{4} \mathrm{OS}$ : C 54.49, $\mathrm{H} 3.05, \mathrm{~N} 12.10$; found C 54.33, H 3.20, N 12.23.

4-氯-1-甲基-3-(4-甲氧基苯基)- $N$ - $\{[4-(3,4$-二氟苯 基)噻唑-2-基]甲基 $\}-1 H$-吡唑-5-甲酰胺(12q)：淡黄色固 体, 产率 50\%. m.p. $129 \sim 131{ }^{\circ} \mathrm{C} ;{ }^{1} \mathrm{H}$ NMR $\left(\mathrm{CDCl}_{3}, 400\right.$ MHz) $\delta: 7.88(\mathrm{~d}, J=2.00 \mathrm{~Hz}, 1 \mathrm{H}), 7.70 \sim 7.76(\mathrm{~m}, 3 \mathrm{H})$, $7.59 \sim 7.63(\mathrm{~m}, 1 \mathrm{H}), 7.43(\mathrm{~s}, 1 \mathrm{H}), 7.18 \sim 7.25(\mathrm{~m}, 1 \mathrm{H})$, $7.01(\mathrm{~d}, J=8.40 \mathrm{~Hz}, 1 \mathrm{H}), 5.01(\mathrm{~d}, J=5.60 \mathrm{~Hz}, 2 \mathrm{H}), 4.24$ $(\mathrm{s}, 3 \mathrm{H}), 3.95(\mathrm{~s}, 3 \mathrm{H}) ;{ }^{13} \mathrm{C} \mathrm{NMR}\left(100 \mathrm{MHz}, \mathrm{CDCl}_{3}\right) \delta$ : $166.4,158.4,155.2,153.2,151.7,149.1,145.0,131.8$, $131.3,129.3,127.0,124.2,122.7,122.3,117.8,115.5$, 113.8, 111.9, 107.2, 56.2, 41.3; ESI-MS m/z: $475[\mathrm{M}+$ $\mathrm{H}^{+}$. Anal. calcd for $\mathrm{C}_{22} \mathrm{H}_{17} \mathrm{ClF}_{2} \mathrm{~N}_{4} \mathrm{O}_{2} \mathrm{~S}$ : C 55.64, $\mathrm{H} 3.61, \mathrm{~N}$ 11.80; found C 55.80, H 3.73, N 11.63.

4-氯-1-甲基-3-(2,4-二氯苯基)- $N$ - $\{[4-(2,4$-二氯苯基) 噻唑-2-基]甲基\}-1H-吡唑-5-甲酰胺(12r)：白色固体，产 率 48\%. m.p. 167 $169{ }^{\circ} \mathrm{C} ;{ }^{1} \mathrm{H} \mathrm{NMR}\left(\mathrm{CDCl}_{3}, 400 \mathrm{MHz}\right)$ $\delta: 7.91(\mathrm{~d}, J=8.80 \mathrm{~Hz}, 1 \mathrm{H}), 7.85(\mathrm{~s}, 1 \mathrm{H}), 7.75$ (s, 1H), 7.53 $(\mathrm{s}, 1 \mathrm{H}), 7.49(\mathrm{~d}, J=2.40 \mathrm{~Hz}, 1 \mathrm{H}), 7.31 \sim 7.34(\mathrm{~m}, 3 \mathrm{H}$, ArH), 5.02 (d, J=5.60 Hz, 2H), $4.28(\mathrm{~s}, 3 \mathrm{H}) ;{ }^{13} \mathrm{C}$ NMR $\left(100 \mathrm{MHz}, \mathrm{CDCl}_{3}\right) \delta: 164.8,158.2,150.4,145.0,135.9$, $135.0,134.3,132.8,132.5,132.2,131.2,130.3,129.8$, 129.2, 128.3, 127.4, 127.1, 119.0, 109.8, 41.5, 41.2; ESI-MS $m / z: 545[\mathrm{M}+\mathrm{H}]^{+}$. Anal. calcd for $\mathrm{C}_{21} \mathrm{H}_{13} \mathrm{Cl}_{5^{-}}$ $\mathrm{N}_{4} \mathrm{OS}$ : C 46.14, H 2.40, N 10.25; found C 46.31, H 2.15, N 10.39 .

\section{辅助材料(Supporting Information) 目标化合物 12a} $12 \mathrm{r}$ 的核磁共振氢谱和碳谱图谱. 这些材料可以免费从 本刊网站(http://sioc-journal.cn/)上下载.

\section{References}

[1] Prakash, T. B.; Reddy, G. D.; Padmaja, A.; Padmavathi, V. Eur. J. Med. Chem. 2014, 82, 347.

[2] Guan, A. Y.; Qin, Y. K.; Wang, J. F.; Li, B. J. Fluorine Chem. 2013, $156,120$.

[3] Li, G. Y.; Qian, X. H.; Cui, J. N.; Huang, Q. C.; Zhang, R.; Guan, H. J. Agric. Food Chem. 2006, 54, 125.

[4] Ohnmacht, S. A.; Ciancimino, C.; Vignaroli, G.; Gunaratnam, M.; Neidle, S. Bioorg. Med. Chem. Lett. 2013, 23, 5351.

[5] Liu, X. H.; Zhao, W.; Shen, Z. H.; Xing, J. H.; Yuan, J.; Yang, G.; Xu, T. M.; Peng, W. L. Bioorg. Med. Chem. Lett. 2016, 26, 3626.

[6] Liu, X. H.; Zhao, W.; Shen, Z. H.; Xing, J. H.; Xu, T. M.; Peng, W. L. Eur. J. Med. Chem. 2017, 125, 881.

[7] Shi, J. J.; Ren, G. H.; Wu, N. J.; Weng, J. Q.; Xu, T. M.; Liu, X. H.; 
Tan, C. X. Chin. Chem. Lett. 2017, 28, 1727.

[8] Wang, B. L.; Zhu, H. W.; Li, Z. M.; Wang, L. Z.; Zhang, X.; Xiong, L. X.; Song, H. B. Pest Manage. Sci. 2018, 74, 726.

[9] Yu, H. B.; Qin, Z. F.; Dai, H.; Zhang, X.; Qin, X.; Wang, T. T.; Fang, J. X. J. Agric. Food Chem. 2008, 56, 11356.

[10] Wang, H. L.; Ruan, L. L.; Chen, Y.; Liu, X. H.; Weng, J. Q. Chin. J. Org. Chem. 2015, 34, 419 (in Chinese). (王海林, 阮铃莉, 陈勇, 刘幸海, 翁建全, 有机化学, 2015, 34, 419.)

[11] Pember, S. O.; Mejia, G. L.; Price, T. J.; Pasteris, R. J. Bioorg. Med. Chem. Lett. 2016, 26, 2965

[12] Mabkhot, Y. N.; Alharbi, M. M.; Al-Showiman, S. S.; Ghabbour, H. A.; Kheder, H. A.; Soliman, S. M.; Frey, W. Chem. Cent. J. 2018, 12,56 .

[13] Althagafi, I.; El-Metwaly, N.; Farahaly, T. A. Molecules 2019, 24, 1741.

[14] Maienfisch, P. L.; Huerlimann, H.; Rindlisbacher, A.; Gsell, L.; Dettwiler, H.; Haettenschwiler, J.; Sieger, E.; Walti, M. Pest Manage. Sci. 2001, 57, 165.

[15] Cao, S. W.; Liu, A. P.; Liu, W. D.; Liu, X. P.; Ren, Y. G.; Pei, H.; Huang, L.; Zheng, X.; Huang, M. Z.; Wu, D. X. J. Heterocycl. Chem. 2017, 54, 3395 .

[16] Ding, C. R.; Pan, Y. Y.; Yin, X.; Tan, C. X.; Zhang, G. F. Chin. J. Org. Chem. 2019, 39, 836 (in Chinese). (丁成荣, 潘亚运, 殷许, 谭成侠, 张国富, 有机化学, 2019，39, 836.)

[17] Ding, C. R.; Pan, Y. Y.; Yin, X.; Tan, C. X. Chin. J. Org. Chem. 2019, 39, 2099 (in Chinese) (丁成荣，潘亚运，殷许，谭成侠，有机化学, 2019, 39, 2099.)

[18] Dai, H.; Li, G.; Chen, J.; Shi, Y. J.; Ge, S. S.; Fan, C. G.; He, H. B. Bioorg. Med. Chem. Lett. 2016, 26, 3818.

[19] Dai, H.; Chen, J.; Li, G.; Ge, S. S.; Shi, Y. J.; Fang, Y.; Ling, Y. Bioorg. Med. Chem. Lett. 2017, 27, 950.

[20] Monicka, J. C.; James, C. J. Mol. Struct. 2014, 1075, 335.

[21] Fulcher, J. M.; Wayment, D. G.; White, Jr, P. M.; Webber III, C. L.; J. Agric. Food Chem. 2014, 62, 2141.

[22] Dai, H.; Ge, S. S.; Guo, J.; Chen, S.; Huang, M. L.; Yang. J. Y.; Sun, S. Y.; Ling, Y.; Shi, Y. J. Eur. J. Med. Chem. 2018, 143, 1066.

[23] Gu, B. Q.; Zhu, W. Q.; Fan, W. Z.; Qian, H.; Liu, J. M.; Zhang, A. Q.; Shen, R. X. Modern Agrochem. 2002, 1,9 (in Chinese). (顾保权，朱伟清，范文政，钱虹，刘建梅，张爱庆，沈荣仙，现
代农药, 2002, 1,9.)

[24] Fan, W. Z.; Gu, B. Q.; Zhu, W. Q.; Zhang, Y. B. Modern Agrochem. 2005, 4, 9 (in Chinese).

(范文政，顾保权，朱伟清，张一宾，现代农药， 2005, 4, 9.)

[25] Hughes, K. A.; Lahm, G. P.; Selby, T. P.; Stevenson, T. M. WO 2004067528, 2004 [Chem. Abstr. 2004, 141, 190786].

[26] Hamaguchi, H.; Kajihara, O.; Katoh, M. J. Pestic. Sci. 1995, 20 173.

[27] Yang, Z. B.; Hu, D. Y.; Zeng, S.; Song, B. A. Bioorg. Med. Chem. Lett. 2016, 26, 1161.

[28] Zhao, W.; Shen, Z. H.; Xu, T. M.; Peng, W. L.; Liu, X. H. J. Heterocycl. Chem. 2017, 54,1751.

[29] Cheng, L.; Shen, Z. H.; Xu, T. M.; Tan, C. X.; Weng, J. Q.; Han, L.; Peng, W. L.; Liu, X. H. J. Heterocycl. Chem. 2018, 55, 946.

[30] Lima, L. M. A.; Barreiro, E. J. Curr. Med. Chem. 2005, 12, 23.

[31] Romagnoli, R.; Baraldi, P. G.; Salvador, M. K.; Preti, D.; Tabrizi, M. A.; Brancale, A.; Fu, X. H.; Li, J.; Zhang, S. Z.; Hamel, E.; Bortolozzi, R.; Basso, G.; Viola, G. J. Med. Chem. 2012, 55, 475.

[32] Wang, Q. M.; Li, H.; Li, Y. H.; Huang, R. Q. J. Agric. Food Chem. 2004, 52, 1918.

[33] Liu, Y. X.; Wei, D. G.; Zhu, Y. R.; Liu, S. H.; Zhang, Y. L.; Zhao, Q. Q.; Cai, B. L.; Li, Y. H.; Song, H. B.; Liu, Y.; Wang, Y.; Huang, R. Q.; Wang, Q. M. J. Agric. Food Chem. 2008, 56, 204.

[34] Dai, H.; Li, Y. Q.; Du, D.; Qin, X.; Zhang, X.; Yu, H. B.; Fang, J. X J. Agric. Food Chem. 2008, 56, 10805.

[35] Yang, Y. Z.; Lin, D. Y.; Fu, C. R.; Zou, X. M. Chin. J. Org. Chem. 2015, 35, 100 (in Chinese). (杨亚喆, 林大勇, 傅翠蓉, 邹小毛, 有机化学, 2015, 35, 100.)

[36] Wang, B. L.; Zhu, H. W.; Ma, Y.; Xiong, L. X.; Li, Y. Q.; Zhao, Y.; Zhang, J. F.; Chen, Y. W.; Zhou, S.; Li, Z. M. J. Agric. Food Chem. 2013, 61, 5483.

[37] Dai, H.; Yao, W.; Fang, Y.; Sun, S. Y.; Shi, Y. J.; Chen, J.; Jiang, G. Q.; Shi, J. Molecules 2017, 22, 2000.

[38] Shi, Y. J.; Ye, L. Y.; Zhong, S. L.; Cao, X. F.; Dai, H.; Hong, Y.; Li, C. J.; Shi, J.; Wu, J. M. Chin. J. Org. Chem. 2017, 37, 1844 (in Chinese).

(石玉军，叶林玉，仲苏林，曹雄飞，戴红，洪宇，李春建，石健， 吴锦明，有机化学, 2017, 37, 1844.)

[39] Shao, L.; Zhou, X.; Zhang, Q.; Liu, J. B.; Jin, Z.; Fang, J. X. Synth. Commun. 2007, 37, 199 